\title{
Micro-usinage nanoseconde UV ou femtoseconde : état de l'art et comparaison
}

\author{
J. Lopez, F. Salin, M.-C. Hernandez et M. Faucon \\ CELIA-PALA, 351 cours de la Libération, bâtiment A33, 33405 Talence cedex, France
}

\begin{abstract}
Résumé : Jusqu'ici les applications liées au micro-usinage étaient principalement réservées aux sources de types $\mathrm{Nd}$ :YAG, pour les matériaux métalliques, ou Excimère, pour les polymères. Les avancées récentes dans le domaine des lasers à impulsions ultra-brèves $(<10 \mathrm{ps})$, en terme de performances, fiabilité et coût, permettent aujourd'hui d'envisager des applications industrielles dans le domaine du micro-usinage. Ces applications tirent profit de la possibilité d'usiner tout type matériau sans diffusion de chaleur hors du volume irradié. Après avoir rappeler les spécificités des interactions avec ces différents types de laser, nous présenterons quelques exemples de réalisations par rayonnement laser UV ou femtoseconde.
\end{abstract}

\section{INTRODUCTION}

Les lasers sont aujourd'hui largement exploités au niveau industriel pour des applications de découpe, soudage et marquage. Les sources utilisées sont pour la plupart des lasers infrarouge $\left(\mathrm{CO}_{2}\right.$ ou YAG) dont la particularité est d'usiner la matière par un processus purement thermique, ce qui implique un échauffement important de la matière et par conséquent des effets collatéraux souvent indésirables : déformation de la surface, fragilisation de la structure, bavures, zone de fusion ou de recuit.... Des lasers de conceptions plus récentes, tels que les lasers femtosecondes ou les lasers UV, permettent aujourd'hui d'usiner de nombreux matériaux par des processus quasi-athermiques - sans effet collatéral. L'objectif de cette présentation est d'expliquer l'intérêt de ce type de lasers et d'en montrer les potentialités dans les domaines du micro-usinage et du marquage à travers quelques exemples de réalisations.

\section{PROCESSUS D'ABLATION AVEC UNE IMPULSION LONGUE ( $>10 \mathrm{ps)}$}

Pour une impulsion longue ( $>10 \mathrm{ps}$ ), le processus est thermique. Le rayonnement - souvent IR ou visible - est ici absorbé par les électrons périphériques (électrons libres pour les métaux, électrons des bandes de conduction ou de valence pour les matériaux non-métalliques), puis il y a conduction thermique et transfert de l'énergie des électrons "chauffés" vers le réseau d'atomes. Cet apport de chaleur localisé, intense et prolongé provoque la fusion puis la vaporisation du matériau. La matière en interaction est principalement expulsée sous forme de vapeurs et de gouttelettes en surfusion que l'on va retrouver en partie sur le pourtour de la zone usinée. Compte tenu de la diffusion thermique, la zone de vaporisation, la zone de resolidification et la zone affectée thermiquement (ZAT) s'étendent au-delà des dimensions du spot laser. La vitesse d'ablation et l'étendue de la ZAT diminuent avec la durée d'impulsion. Ce processus thermique favorise un usinage rapide mais il s'accompagne de nombreux effets indésirables. Une onde acoustique, résultant de l'élévation brutale de température et de l'impulsion mécanique liée au départ de matière, se propage de la surface vers le corps du matériau provoquant décohésion, écaillage, fissuration et contrainte mécanique dans le matériau. L'ampleur de cette onde est liée au volume vaporisée et à la quantité de chaleur déposée par le laser. Le plasma engendré par le front de montée de l'impulsion laser accroît momentanément la réflectivité de la cible, ce qui provoque le découplage laser-cible et donc une perte d'efficacité du procédé [1]. 
Cas particulier de la photoablation: Ce processus de décomposition photochimique concerne principalement l'interaction entre un rayonnement ultraviolet et un matériau moléculaire (polymère, composé organique...). L'énergie du photon étant comparable à celle des liaisons covalentes du matériau cible, l'absorption d'un seul photon par un électron de valence permet de "briser" une liaison moléculaire. Au-delà d'un certain nombre de liaisons brisées par unité de volume, la matière explose et est éjectée sous forme de gaz et de fragments moléculaires. Cette expulsion de matière à vitesse supersonique permet d'évacuer l'excès d'énergie sous forme d'énergie cinétique. Les détériorations thermiques et mécaniques de la cible sont donc négligeables. L'épaisseur ablatée varie de 0,1 à $5 \mu \mathrm{m}$ selon la longueur d'onde, la fluence et le coefficient d'absorption du matériau, ce qui permet de maîtriser précisément la profondeur usinée. La différence de seuil d'ablation entre certains matériaux de nature différente - métal et polymère par exemple - peut être mis à profit pour réaliser une ablation sélective (exemple d'application : ablation de la gaine externe en plastique sur un fil métallique ou fibre optique). La décomposition photochimique, a priori athermique, va entrer plus ou moins en compétition avec des phénomènes de relaxation radiatifs ou non-radiatifs pouvant eux conduire à un échauffement local du matériau. D'une manière générale, plus un polymère est absorbant plus il sera décomposé, moins il y aura d'effet thermique, plus l'épaisseur ablatée sera faible et meilleur sera l'état de surface. Des effets thermiques et/ou collatéraux peuvent donc apparaître sur les matériaux non-fortement absorbants dans l'UV.

Pour des matériaux non organiques le rayonnement UV présente deux avantages par rapport au rayonnements IR ou visible :

- Le couplage laser-plasma est moins prononcé en UV qu'en IR ou visible [2]

- Le coefficient d'absorption des matériaux est en général plus élevé en UV qu'en IR ou que dans le visible; l'épaisseur mise en jeu et les effets collatéraux sont moins marqués en UV.

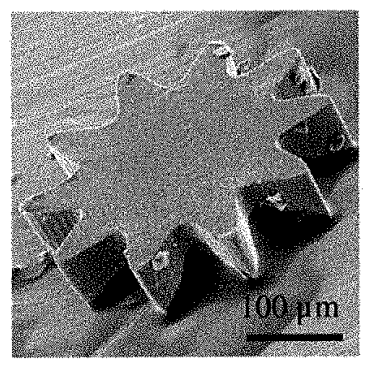

Micrographie 1.

Découpe sur polymère $\varnothing 400 \mu \mathrm{m}$

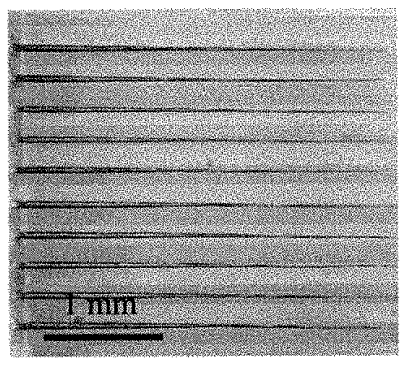

Micrographie 2.

Perçages sur polymère (PMMA) $\emptyset 30 \mu \mathrm{m}$ Prof. $3 \mathrm{~mm}$

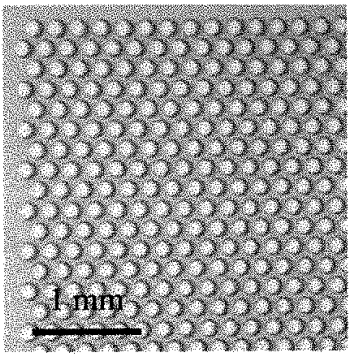

Micrographie 3.

Matrice de trous (PI)

$\varnothing 150 \mu \mathrm{m}$ Ep. $250 \mu \mathrm{m}$

La mise en forme du faisceau est déterminante sur la qualité et la reproductibilité finale de l'usinage. A ce niveau plusieurs paramètres sont à prendre en compte : focalisation, ouverture du faisceau, fluence, polarisation, gaz de couverture... Les lasers Excimères ayant un lat ge faisceau et un faible de taux de répétition, il est préférable de travailler en projection de masque : statique, balayage / déplacement de la cible, balayage de masque, ou balayage avec recouvrement de masque synchronisé [3]. La technique de projection de masque permet en outre d'obtenir un profil d'intensité flat top et donc un meilleur contraste sur la cible (micrographie 1). Ce meilleur contraste se traduit par des contours francs [3], une meilleure conicité et une profondeur maximale usinée accrue (rapport profondeur/diamètre supérieur à 300 sur certains polymères, voir micrographie 2) [4]. A noter que le masque utilisé en 
projection absorbe une partie non négligeable de l'énergie initiale du faisceau (transmission $<20 \%$ ), ce qui peut conduire à des effets inverses à ceux recherchés et donner des bords irréguliers [4]. La technique de projection de masque est utilisée pour la gravure de surface (micrographie 3 ), le micro perçage profond, et l'usinage de micro systèmes combinant trous, canaux, réservoirs, engrenages ou autres micro objets $[1,4]$.

Avec les lasers à solides (solid-state laser) à 355 ou $266 \mathrm{~nm}$, qui ont une énergie par impulsion plus faible et un taux de répétition bien plus élevé ( 3 à $100 \mathrm{kHz}$ ), il est préférable de travailler en écriture directe. Le laser est alors couplé avec une table motorisée ou une tête à déflection optique. Cette configuration offre une grande souplesse d'utilisation, en revanche le contraste est moins marqué qu'en projection de masque. L'écriture directe est utilisée pour la micro découpe, le microperçage, et pour quelques applications de gravure [3].

\section{PROCESSUS D'ABLATION AVEC UNE IMPULSION ULTRA-BREVE ( $<500$ fs)}

Le processus d'ablation avec une impulsion ultra-brève (femtoseconde) met en jeu des phénomènes non linéaires [1]. La nature athermique de ce processus est liée à la brièveté des impulsions $\left(10^{-13} \mathrm{~s}\right)$ et à leur forte intensité $\left(10^{12}\right.$ à $10^{14} \mathrm{Watt} / \mathrm{cm}^{2}$ une fois focalisé). Le " chauffage » des électrons par absorption multiphotonique fait appel à un processus de type Bremsstrahlung inverse. Les premiers électrons éjectés (électrons libres) vont ensuite transmettre leur énergie aux autres électrons du réseau d'atomes par chocs et provoquer une avalanche d'ionisation, laquelle sera suivie d'une expulsion de matière. Le terme athermique est un peu abusif car la température électronique dans le panache peut atteindre plusieurs milliers de Kelvin, cependant il décrit bien l'absence d'effet thermique collatéral. En effet, le temps caractéristique de diffusion des électrons excités et de transfert de l'énergie vers le réseau d'atome (10 ps) est bien supérieur à la durée d'impulsion femtoseconde. La matière est donc éjectée avant qu'il n'y ait diffusion de la chaleur hors du volume irradié. La ZAT et la zone fondue existent mais elles sont considérablement réduites par rapport à une impulsion longue. Indépendamment du coefficient d'absorption du matériau à la longueur d'onde considérée, les fortes intensités atteintes permettent l'ablation de tout type de matériau. Des expériences comparatives indiquent que le fait de passer de 100 à $10 \mathrm{fs}$ améliore sensiblement les précisions latérale et verticale [1]. Les fluences utilisées en micro usinage varient de 0,2 à $50 \mathrm{~J} / \mathrm{cm}^{2}$ selon la qualité et la vitesse d'usinage recherchées. Les vitesses d'ablation, comprises entre 0,1 et $1 \mu \mathrm{m} / \mathrm{pls}$ selon les matériaux, sont plus faibles comparativement aux lasers d'usinage traditionnels.

Pour des impulsions ultra-brèves, l'absorption et l'expulsion de matière ne sont pas simultanées, il n'y a donc pas d'écrantage de la cible par le panache d'ablation [5]; cependant à forte intensité - cas du micro-usinage - le faisceau focalisé provoque un claquage de l'air ambiant au niveau du plan focal. Ce plasma modifie la structure du faisceau et il est susceptible de provoquer des effets non-linéaires aléatoires (déformation du profil énergétique, autofocalisation ou défocalisation, filamentation) souvent nuisibles à la qualité de l'usinage. Pour éviter la formation du plasma et minimiser ces effets indésirables il convient de travailler sous vide primaire [6] ou d'utiliser un jet de gaz inerte (hélium, argon). L'expérience montre que l'utilisation d'un gaz d'assistance tel que l'hélium améliore la rugosité des parois internes des zones usinées [5]. La majorité des applications peuvent être traitées en écriture directe - en utilisant une tête à déflection optique et une optique de focalisation de type $f(t h e ̂ t a)$. L'utilisation d'une optique diffractive adaptée permet d'optimiser le contraste ou d'obtenir un profil d'intensité spécifique sans être pénalisé par la formation d'un plasma au-devant de la cible [7] et de minimiser les pertes d'énergie 
(transmission $\sim 80 \%$ ) [5]. Ce type d'optique, dont le design est réalisé sur mesure, donne de bons résultats en pratique en terme de profondeur usinée, circularité, homogénéité et rugosité des parois [5], mais reste onéreux et manque de souplesse puisque le profil d'intensité et la géométrie du faisceau sont fixés.

Dans le cas d'un usinage profond, une polarisation circulaire diminue les effets anisotropes et améliore sensiblement la géométrie du motif usiné (meilleure circularité d'un perçage par exemple) [7]. Par ailleurs, il est sera préférable d'utiliser un faisceau de faible ouverture pour un usinage profond.

L'absence d'accumulation de chaleur dans la cible autorise des usinages jusqu'ici irréalisables tels :

- la découpe de motifs favorisant le confinement de la chaleur tels des grilles ou des barreaux de faible section (micrographies 4,5 et 6 )

- la découpe de films ou feuillets multicouches hétérogènes sans délamination

- l'usinage d'un métal sans formation de bavures (micrographie 5)

- l'usinage de verres, céramiques (micrographie 7) ou matériaux ultra-durs sans formation de micro fissures

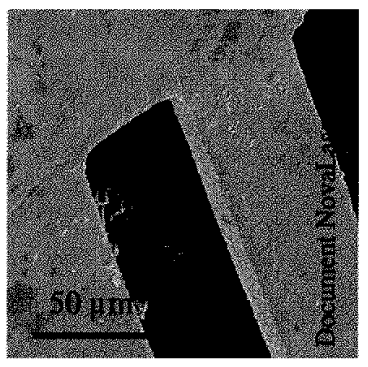

Micrographie 4.

Grille en or

Pas $50 \mu \mathrm{m}$ Ep $35 \mu \mathrm{m}$

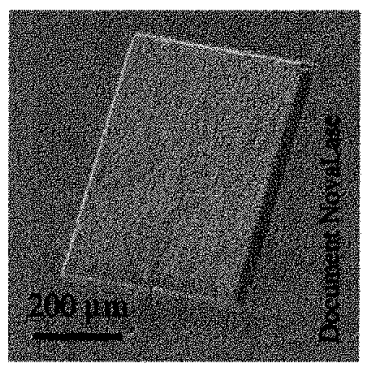

Micrographie 7.

Gravure de surface sur $\mathrm{SiC}$ côté $400 \mu \mathrm{m}$

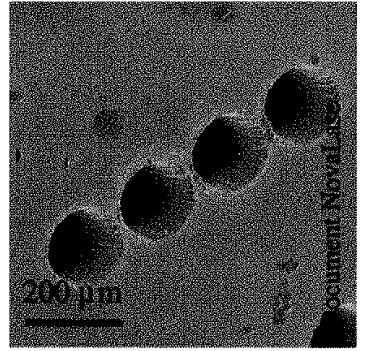

Micrographie 5.

Perçages sur acier inox

Ø $170 \mu \mathrm{m}$ Prof. $250 \mu \mathrm{m}$

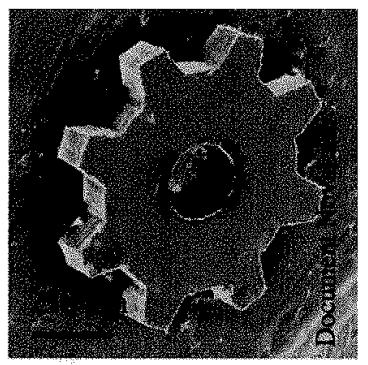

Micrographie 8.

Micro engrenage (Kapton)

Diamètre $800 \mu \mathrm{m}$

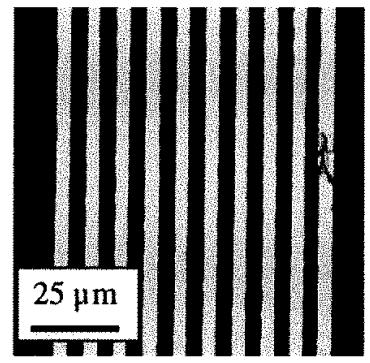

Micrographie 6.

Grille en or

Pas $10 \mu \mathrm{m}$ Ep. $25 \mu \mathrm{m}$

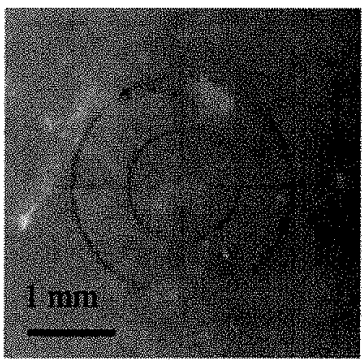

Micrographie 9.

Marquage interne sur $\mathrm{SiO}_{2}$ Prof. $500 \mu \mathrm{m}$

Les applications des lasers femtosecondes concernent principalement le micro perçage, le marquage et la micro découpe (micrographie 8). Dans le cas du marquage la gravure est propre, homogène et non-évolutive. La gravure étant très fine (trait $10-50 \mu \mathrm{m}$ ), il 
est possible de réaliser des marquages invisibles à l'œil nu. Il est montré que ces lasers offrent également de nouvelles potentialités en médecine: découpe de stent [5], chirurgie réfractive [8], ou encore découpe de dentine [9].

Marquage interne : En utilisant un faisceau fortement convergent, il est possible d'usiner sous la surface d'un matériau transparent à une profondeur contrôlée pouvant aller de quelques dizaines de microns à quelques millimètres (micrographie 9). L'usinage a lieu uniquement au point focal, là où la densité de puissance est supérieure au seuil du matériau. Les paramètres laser et la nature du matériau vont conditionner le type d'usinage : variation d'indice, création de centres colorés, écaillage ou vaporisation. Cette modification locale de la matière s'accompagne de phénomènes thermiques et/ou mécaniques susceptibles de provoquer des effets collatéraux, dont l'ampleur est liée à la durée d'impulsion. L'utilisation d'impulsions ultra-brèves permet de minimiser les contraintes induites et d'obtenir une finesse de trait inégalable avec une source laser conventionnelle. Les applications en développement concernent la gravure de guides optiques ou de mires, la création de défauts calibrés, la lutte anti-contrefaçon ainsi que diverses opérations en chirurgie réfractive (laser intrastromal [4]).

\section{CONCLUSION}

Les lasers femtosecondes affichent clairement des potentialités intéressantes dans les domaines du micro-usinage et du marquage sur la plupart des matériaux. Le principal avantage est l'absence de diffusion de chaleur pendant l'usinage. L'arrivée de sources industrielles fiables, et notamment de lasers femtosecondes pompés diodes, devrait permettre l'émergence de procédés industriellement viables dans les années à venir sur certaines applications niches.

Sur certains matériaux - dont de nombreux polymères absorbants et certains types de céramiques - les lasers UV donnent des résultats proches de ceux obtenus avec des impulsions ultra-brèves mais avec des temps d'usinage et coût de process plus intéressants. Pour des faisceaux larges de puissance élevée (Excimère) on préférera travailler en projection de masque, voire en multifaisceaux ; pour des lasers de plus faible puissance ( $<10$ Watt) et de taux de répétition élevé ( $>3 \mathrm{kHz}$ ) on préférera travailler en écriture directe en utilisant une tête à déflection optique. Ce dernier montage offre une grande souplesse d'utilisation.

\section{Remerciements}

A l'Union Européenne et au Conseil Régional d'Aquitaine pour le co-financements des équipements scientifiques utilisés pour ces travaux de recherche.

\section{Références}

[1] Lenzner et al., Appl.Phys.A, Vol. 68, p. 369-371 (1999)

[2] Varel et al., Appl.Phys.A, Vol. 65, p. 367-373 (1997)

[3] N. Rizvi et M. Gower, Site Internet de Exitech Limited, www.exitech.co.uk (1999-2001)

[4] J. Lopez, S. Lazare et F. Weisbuch, Proceeding Europto Series, Vol. 3822, p.77-82 (1999)

[5] C. Momma, S. Nolte. B.N. Chichkov. F.V. Alvensleben et A. Tünnermann, Appl.Surf.Sci., Vol. 109-110, p.15-19 (1997)

[6] S. Nolte, G. Kamlage, F. Korte, T. Bauer, T. Wagner, A. Ostendorf, C. Fallnich et H. Welling, Adv.Eng.Mat., Vol. 2 No. 1-2, p.23-27 (2000)

[7] H.K. Tonshoff, C. Momma, A. Ostendorf, S. Nolte et G. Kamlage, Proceeding of the Laser Materials Processing Conference, ICALEO'98, p.A28-A37, Vol. 1 (1998)

[8] H. Lubatschowski et al., Graefe's Arch.Clin.Ophtalmol. (2000)

[9] P. Kohns, P. Zhou et R. Stoermann, J.Laser.Appl., Vol. 9 Issue 3, p.171-174 (1997) 